\title{
SPATIAL ATTENTIONAL SHIFTS: IMPLICATIONS FOR THE ROLE OF POLYSENSORY MECHANISMS
}

\author{
Henry A. Buchtel* and Charles M. Butter \\ Ann Arbor VA Medical Center and the University of Michigan \\ (Received 6 January 1987; accepted 17 July 1987)
}

\begin{abstract}
Simple reaction times to lateralized visual (Experiment 1) or auditory (Experiment 2) targets were studied in normal subjects. The targets were preceded by a visual or auditory cue located on the same (valid cue), or opposite (invalid cue) side as the subsequent target, or on both sides (neutral cue), with one of four cue target intervals. The validity of visual and auditory cues influenced the speed of response to the visual target but not to the auditory target. It is hypothesized that crossmodal cueing of spatial position works only with modalities for which a movement (e.g. saccade) leads to improved sensory analysis.
\end{abstract}

\section{INTRODUCTION}

SPATIALLY-DIRECTED attention has been demonstrated in several experimental situations [5, 10,157 , and has been shown to affect both visual detection $[2,12,14]$ and the speed of responding [10]. Most studies of spatially-directed attention have employed visual cues to control the direction of attention to visual targets. Very few studies (described below) have employed spatial cues in modalities different from those of the (visual or non-visual) target. The possibility that attention can be spatially directed under these conditions is supported by neurophysiological findings that the brain structures implicated in directing attention spatially receive convergent input from several sensory modalities. Moreover, contralateral neglect results from unilateral removal of these polysensory structures [cf. 4]. According to these findings, then, one would expect that spatial cues in various modalities would also be effective in shifting attention to stimuli in any particular sensory modality. POSNER [10] reported findings that are apparently contradictory to this prediction: visual cues that produce costs and benefits in reaction times ( $\mathrm{RTS}$ ) to visual targets do not produce costs or benefits in RTs to auditory targets or to tactile targets, except when the subjects are required to discriminate between the tactile targets. However, in these experiments the visual cues were symbolic (arrows in the center of this display pointing to the left or right) rather than spatial. Moreover, Posner did not report the effects of non-visual cues on RTs to visual targets.

The experiments reported here were undertaken to further test the effects of spatial cues on speed of reaction to target stimuli in the same and different modalities. Experiment 1 compared the effects of visual and auditory spatial cues on RTs to visual stimuli; Experiment 2 compared the effects of the same spatial cues on R Ts to auditory stimuli. On the basis of the

\footnotetext{
*To whom correspondence should be addressed at: Psychology (116B), Ann Arbor V.A. Medical Center. 2215 Fuller Road. Ann Arbor. MI 48105. U.S.A
} 
neurophysiological and neurobehavioral findings cited above, we predicted that spatial cues in a sensory mode different from that of the target stimuli would be as effective in shifting attention as spatial cues in the same sensory mode as that of the target stimuli.

\section{EXPERIMENT 1}

\section{Methods}

Subjects. The subjects were 10 men, 2251 yr of age (median age $=25 \mathrm{yr}$ ). Their vision was normal or corrected to normal. All were right-handed by self-report. Half of the subjects were paid $(\$ 5.00 / \mathrm{hr})$ at the end of the experiment for their services; the remaining subjects volunteered without payment (this difference had no effect on the results). Three of the subjects were aware of the purpose of the experiment prior to testing; the others were naive.

Apparatus and procedures. The subjects sat in a sound-attenuated room and faced a black tangent screen lat a distance of $57 \mathrm{~cm}$ ) on which the stimuli were presented. The visual targets were provided by two, red light-emitting diodes (LEDs), one located $10^{\prime}$ to the left, the other $10^{\prime \prime}$ to the right of the center of the screen. The visual cues consisted of four LEDs arranged in a square pattern around each of the visual targets, as shown in Fig. 1. The cueLEDs were approximately 1.8 from the target LED. The auditory cues were provided by bursts of white noise from two small loudspeakers located behind the visual stimuli. The intensity of the auditory stimuli was set at a level established in pilot experiments in which subjects were asked to match the intensity of the auditory stimuli to that of the visual cues. A yellow/green LED, which was continuously illuminted during the testing, was lucated in the center of the sereen and served as a fixation spot (see Fig. 1).

\section{$\because \because \quad \because$}

FIci. I. Stimulus array. Each dot represents one of the LEDs. Speakers are located behind the LED arrays on the left and right. The center LED is yellow/green and served as fixation spot. The other LEDs were red. The two LEDs on the same level as the fixation spot were targets; the four LEDs forming a square around the target came on together and served as the visual cue.

During testing the subjects sat with their chins resting in a molded support. White noise from a speaker located in the testing room was continuously presented during testing to mask outside noises. For subjects who were unfamiliar with the situation. cye position was monitored with a seleral reflectance method (Eye-Trak) in the first session. The subjects initiated each trial by first fixating the central spot and then depressing a microswitch with the right thumb. After a $0.52 \mathrm{sec}$ interval, either one cue was presented on the left or right ( $69 \%$ of trials) or (on neut ral trials) hoth cues were presented simultaneously $(21 \%$ of trials). On $10 \%$ of the trials, no target was presented after the 50 msec cue; these "catch trials" were presented in order to ensure that the subjects responded to the target and not to the passage of time after the cue appeared. The duration of the cues was always 50 msec. The target light then appeared on the left or right side. The time between the onset of the cue and of the target, or stimulus onset asynchrony (SOA), was $50,150.400$ and $1000 \mathrm{msec}$. The subjects released the switch when they detected the target. The opening of the switch terminated the target. If the subject did not respond within $600 \mathrm{msec}$ of the target onset, a "non-response" was recorded. On $80 \%$ of the trials with a single lateralized cue, the target was presented on the same side as the cue ("valid cue trial"), on $20 \%$ of the single-cue trials. the target was presented on the oppositc side ("invalid cue trial"). Responses within 100 msec of target onset were classified as "anticipations" and discarded. If a subject made 15 anticipations and or responses on catch trials, the session was halted, the subject was told to be more careful and the trials were repeated. The different types of trials (valid neutral, invalid. catch trials) were presented in a pseudo-random order. The order of presentation of the targets on the left and right sides and the SOAs was also randomized.

Prior to the first session the subjects were instructed in performance of the task. They were also told to release the microswitch as quickly as possible when the target appeared and to expect the target to appear predominantly on the side where the single cue was presented.

The first session was a practice session in which 696 trials were presented with each cue but not recorded for later analysis. The remaining four sessions. each $6070 \mathrm{~min}$. started with 16 warm-up trials that were not recorded: they were followed by two blocks of 696 trials each. In one block, the visual cue was presented; in the other block the auditory cue was presented. The order of presentation of the two cues was counterbalanced in an ABBA order. Onehalf of the subjects started the first session with the visual cute; the other subjects started the first session with the auditory cue. 
Results

The median RTs of individual subjects were averaged over sessions and analyzed with a repeated-measures, mixed-design ANOVA which included the order in which the subjects were tested with the visual and auditory cues (the between-subjects factor) and four withinsubjects factors: cue modality, cue validity, SOA and the field in which the target was presented. RTs (averaged over subjects) at particular combinations of factors were compared with one-tail or two-tail $t$-tests, using the mean-square error term for the appropriate interaction term and adjusting the confidence limits for the number of comparisons. On the basis of pilot work in which the neutral cue produced effects indistinguishable from those of the invalid cue, we predicted a validity effect (i.e. faster RTs with valid cues than invalid cues) but not costs. In Fig. 2 the median RTs, a veraged over subjects, are plotted to show the effects of three of the within-subjects' factors (cue modality, cue validity and SOA), collapsed over order of cue testing, since neither this factor nor its interactions with other factors were significant sources of variance.
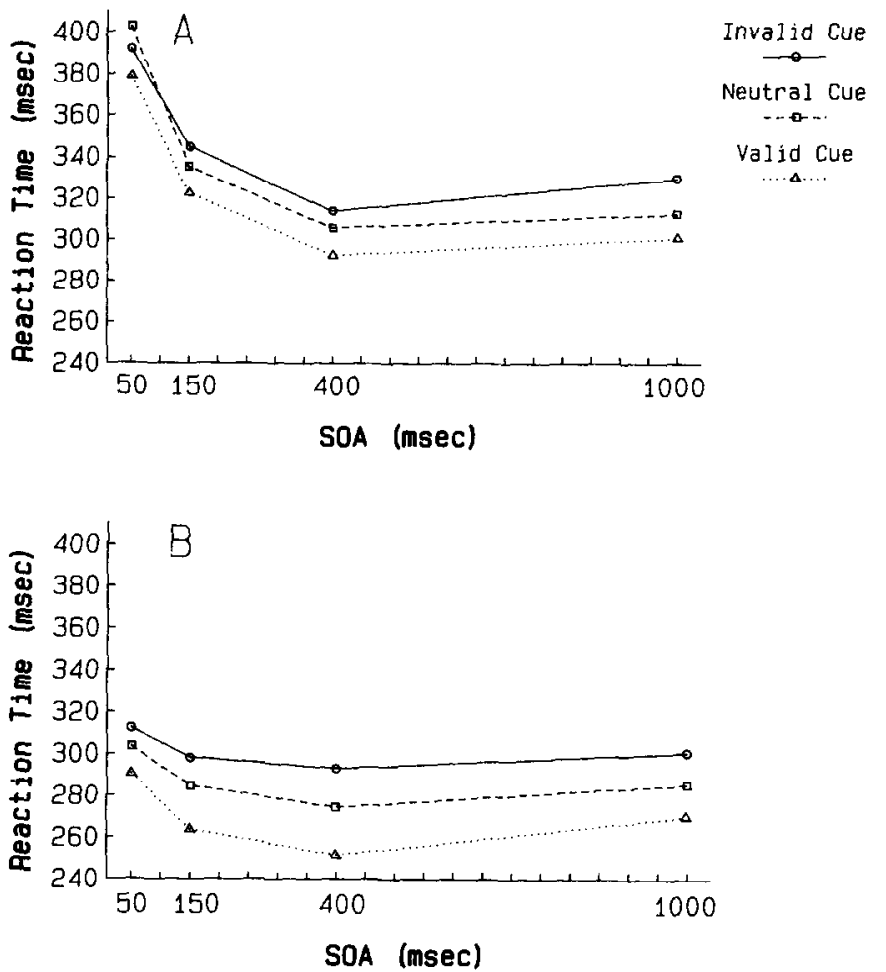

Fici. 2. Median reaction times to the visual target for the four Stimulus Onset Asynchronies (SOAs) and the three validity conditions. (A) Visual cues. (B) Auditory cues.

Considering first the predicted effects of the visual cue, its validity had consistent and expected effects at all SOAs: RTs on trials with valid visual cues were shorter than RTs on trials with invalid cues (see Fig. 2A). These effects of validity were supported by the results of $t$-tests using confidence levels adjusted for multiple one-tail comparisons $(P<0.012)$; 
comparisons at each SOA yielded significant RT differences (see Table 1). It will also be noted that there were costs and benefits of the visual cue al all SOAs except al $50 \mathrm{msec}$, where the average RT on neutral cue trials was longer than the average RT on invalid-cue trials. However, only two of these cost/benefit effects were statistically significant by two-lail $t$-tests (see Table 1). Furthermore, the auditory cue, like the visual cue, had consistent effects upon performance; at all SOAs the valid auditory cue was associated with shorter $\mathrm{RT}$ s than those associated with the invalid auditory cue (see Fig. 2B). These cue validity effects were highly significant when evaluated by one-tail $t$-tests (see Table 1 ). In addition, the auditory cue also had significant benefits at the first three SOAs; at the $1000 \mathrm{msec}$ SOA the benefit of the auditory cue narrowly missed significance (see Table 1). Only the cost at the $400 \mathrm{msec}$ SOA was statistically significant; at the $50 \mathrm{msec}$ SOA the effect approached significance and at the $150 \mathrm{msec}$ SOA there was a non-significant trend (see Table 1).

Table 1. Validity effects and cost benefit analysis of Experiment 1

\begin{tabular}{|c|c|c|c|c|c|c|c|c|}
\hline \multirow{4}{*}{$\begin{array}{l}\text { SOA (msec): } \\
\text { I value: } \\
P \text { value: }\end{array}$} & \multicolumn{5}{|c|}{$\begin{array}{l}\text { Validity effect: } \mathrm{RT}_{\text {(invilid) }} \cdot \mathrm{RT}_{\text {(wilidi }}{ }^{*} \\
\text { Visual cues }\end{array}$} & \multicolumn{2}{|c|}{ Auditory cues } & \multirow[b]{2}{*}{1000} \\
\hline & 50 & 150 & 400 & 1000 & 50 & \multicolumn{2}{|c|}{$150 \quad 400$} & \\
\hline & $3.033^{+}$ & 5.14 & $4.98 \%$ & $6.67 \%$ & $5.00 \%$ & $7.91 \%$ & 9.36 & 7.04 \\
\hline & $<0.01$ & $<0.0005$ & $<0.0005$ & $<0.0005$ & $<0,0005$ & $<0.00015$ & $<0.0005$ & $<0.0005$ \\
\hline \multicolumn{9}{|c|}{ Costs: $R T_{\text {(innalict) }}-R T_{\text {(ncutral }}{ }^{\dagger}$} \\
\hline SOA (msec): & 50 & 150 & 400 & 1000 & 50 & 150 & 400 & 1000 \\
\hline 1 value: & 2.50 & 2.94 & 1.90 & $3.88 \%$ & 3.388 & 2.16 & 4.27 & $3.51 \$$ \\
\hline$P$ value: & $<0.02$ & $<0.01$ & $<0.10$ & $<0.005$ & $<0.01$ & $<0.10$ & $<0.005$ & $<0.01$ \\
\hline \multicolumn{9}{|c|}{$\begin{array}{l}\text { Benefits: } \mathrm{RT}_{(\text {incurall }}-\mathrm{RT}_{\text {(atikit }}{ }^{\dagger} \\
\text { Visual cues }\end{array}$} \\
\hline SOA (msec): & 50 & 150 & 400 & 1000 & 50 & 150 & 400 & 10000 \\
\hline I value: & $5.53 \%$ & 2.20 & 3.07 & 3.07 & $3.69+$ & $4.72 \div$ & $5.30+$ & $3.53 \$$ \\
\hline$P$ value: & $<0.001$ & $<0.10$ & $<0.02$ & $<0.02$ & $<0.005$ & $<0.005$ & $<0.005$ & $<0.01$ \\
\hline
\end{tabular}

As seen in Fig. 3, the magnitude of the validity effects of the auditory cue was somewhat larger than that of the visual cue; correspondingly the cue validity $\times$ cue-modality interaction was significant $(F=3.83 ; d f=2 / 16 ; P=0.044)$. However, neither the validity effects, costs, nor benefits of the two cues were significantly different when evaluated by two-tail $t$-tests $(P<0.4 ; P<0.7$, respectively). Since the three-way interaction of cue modality, validity and SOA did not attain statistical significanee, there were apparently no differential effects of the two eues on costs or benefits at particular SOAs.

It is clear from inspection of Fig. 2 that SOA had an effect not only on performance with the visual cue, but also a somewhat smaller effect on performance with the auditory cue; correspondingly, the ANOVA disclosed a highly significant effect of SOA $(F=13.42$; $d f=3 / 24 ; P<0.0001)$. To evaluate the effect of SOA in the visual cue condition. RTs were averaged over all three validity conditions (valid, neutral and invalid). The results of this analysis showed that the RT decrements over the first three SOAs were highly significant when evaluated by two-tail $t$-tests with the confidence level adjusted $100.02(50 \mathrm{vs} 150 \mathrm{msec}$ 


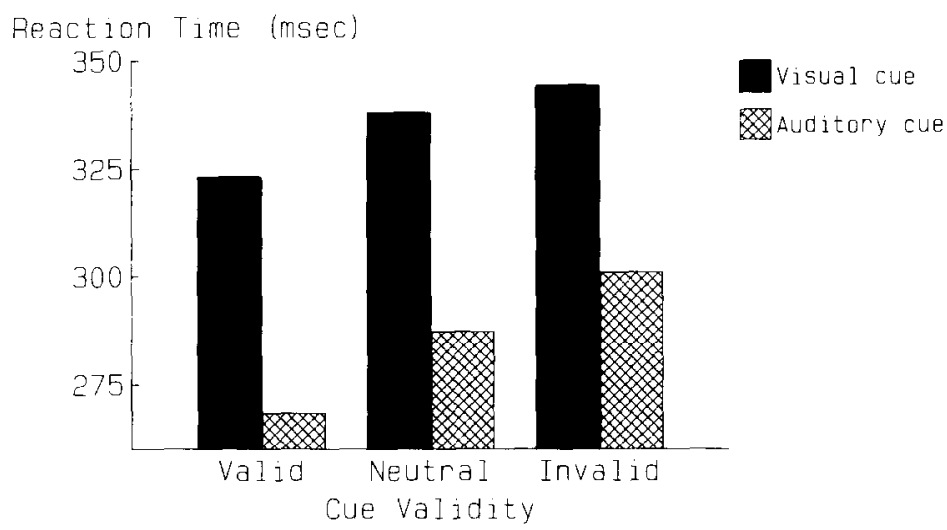

Fic. 3. Effect of modality of cue and cue validity on reaction times to visual targets. averaged across SOAs.

SOA: $t=13.07 ; d f=9 ; P<0.001 ; 150$ vs 400 msec SOA: $t=7.00 ; d f=9 ; P<0.001)$, but the apparent RT increment from $400-1000 \mathrm{msec}$ did not attain significance $(t-2.50 ; d f=9$; $P<0.05)$.

As was the case when the visual cue was presented, when the auditory cue was presented RTs decreased over the first three SOAs and then increased somewhat from $400-1000 \mathrm{msec}$. RTs, averaged over the three validity conditions of the auditory cue, were compared as successive SOAs by $t$-tests (confidence level adjusted to 0.02 or less). The decrement from 50 to $150 \mathrm{msec}$ was significant $(t=4.56 ; d f=9 ; P<0.01)$, as was the increment from 400 to $1000 \mathrm{msec}(t=2.87 ; d f=9 ; P<0.02)$, but the decrement from 150 to $400 \mathrm{msec}$ approached significance $(t=2.09 ; d f=9 ; P<0.1)$.

Inspection of Fig. 2 discloses that the effect of increasing SOA from 50 to $400 \mathrm{msec}$ was smaller when the auditory cue was presented than it was when the visual cue was presented. This differential effect of the cues on the slopes of the SOA functions was reflected in the ANOVA as a highly significant cue modality $\times$ SOA interaction $(F=34.32 ; d f=3 / 24$; $P<0.0001)$. Analysis of this interaction revealed that the decrement associated with the visual cue from $50-150 \mathrm{msec}$ was significantly larger than the decrement associated with the auditory cue over the same SOA range $(t=3.09 ; d f=24, P<0.01)$ but neither the RT decrements from $150-400 \mathrm{msec}$ nor the increments from $400-1000 \mathrm{msec}$ were reliably different from each other in the two cue conditions.

In addition to the effects reported above, there was a main effect of cue modality (for visual cue, mean $\mathrm{RT}=349.9$; for auditory cue, mean $\mathrm{RT}=324.6)(F=32.97 ; d f=1 / 8 ; P=0.0004)$ and field of target presentation $(F=12.02 ; d f=1 / 8 ; P=0.009)$ on RTs. The lalter effect was due to slightly but consistently shorter RTs to targets presented in the right hemifield $(\bar{X}=308.6 \mathrm{msec})$ than RTs to targets presented in the left hemifield $(\bar{X}=313.0 \mathrm{msec})$. This effect was probably due to subjects' use of the right hand in responding $[1,9]$. However, field of target presentation did not interact significantly with any other factor. Cue order interacted significantly with cue validity $(F=5.27 ; d f=2 / 16 ; P<0.017)$; this effect was due to the greater overall cue validity benefit for the group that was tested first with the visual cue compared to the benefit showed by the group that was tested first with the auditory cue. Finally, the cue validity $\times$ SOA interaction was also significant $(F=3.42 ; d f=6.48$; 
$P=0.0069$ ), reflecting the finding that the overall costs and benefits (ignoring cue modality) were bigger at longer SOAs than at shorter SOAs.

\section{Discussion}

The results obtained in this experiment confirmed our hypothesis that a non-visual spatial cue, in this case an auditory cue, would be as effective as a visual spatial cue in shifting attention to a visual target. In fact, the validity effects of the auditory cue were somewhat larger than those of the visual cue. Although the cue validity-cue modality interaction was statistically significant, the finding that neither the overall validity effects nor the component costs and benefits of the two cues differed significantly indicates that no one component was crucial for the somewhat more potent effect of the auditory cue. It is possible that a difference between the two cues in intensity or salience, rather than a genuine modality difference in the attentional properties of the two cues, was responsible for the greater attentional effect of the auditory cue.

The finding that RTs associated with the auditory cue were faster than those associated with the visual cue could have been due to a greater general alerting effect of the auditory cue. Alternatively, this finding may reflect an RT advantage when the cue modality differs from that of the target stimulus compared to the situation in which the cue and target are in the same modality. That is, the subjects may first have to inhibit their response to the cue; this inhibition may require effort and time to dissipate and thus increase RTs. Since a similar effect was also found in Experiment 2, this issue will be discussed after those results are presented.

Another effect found in Experiment 1, and one that may have a similar cause, was the greater RT decrement from $50150 \mathrm{msec}$ associated with the visual cue than the comparable RT decrement associated with the auditory cue. This difference may reflect a more rapid development of alerting effects in the auditory system than in the visual system. Alternatively, it may be due to an intramodal inhibitory effect, referred to above, which would be expected to be maximum at the shortest SOAs. It is also possible that the smaller RT decrement associated with the auditory cue may be duc to a floor effect in this modality. Of course, these alternative interpretations of this finding are not mutually exclusive. Since a differential effect of cue modality on RT decrements associated with the shorter SOAs was also found in Experiment 2, this issue will be discussed later.

\section{EXPERIMENT 2}

Experiment 2 was undertaken in order to further test the hypothesis that cues and targets in different modalities are as effective as cues and targets in the same modality in shifting attention spatially. In this experiment, the effects of the same visual and auditory cues used in the previous experiment were assessed on RTs to an auditory target stimulus.

\section{Methods}

Subjects. The subjects were eight men, 22-51 yr of age (median age $=25.5 \mathrm{yr}$ ), half of whom had served as subjects in Experiment 1. The test-naive subjects, like the experienced ones, were right-handed by self-report; their vision was normal or corrected to normal. The new subjects were paid $(\$ 5.00 / \mathrm{hr})$ at the end of the experiment; the remainder volunteered without payment. The experienced subjects were aware of the purpose of the experiment before testing began; the test-naive subjects were not.

Apparatus and procedures. The apparatus, procedures and cue stimuli were identical to those used in Experiment 1, except that the target stimulus was now a $50 \mathrm{msec}$ hurst of white noise that differed in frequency composition from the auditory cue in that high-frequency components were filtered out. The auditory target stimuli were produced by 
the same speakers that provided the auditory cues. The subjects demonstrated in practice sessions that they could identify the direction from which the auditory targets came (left vs right) and could distinguish them from the auditory cues on the basis of their perceived pitch.

\section{Results}

As in Experiment 1, the median RTs were averaged over sessions; they were then analyzed with a repeated-measures ANOVA that included two between-subjects factors-order of testing with the two cues and experience (test-naive vs experienced subjects) and four withinsubjects factors: cue modality, cue validity, SOA and side of presentation of the auditory target.

As shown in Fig. 4, neither cue produced the validity effects found in Experiment 1; consequently, there were no costs or benefits. The only effect of cue validity was a $2 \mathrm{msec}$ advantage of neutral cues over valid cues, which approached significance $(F=3.68 ; d f=2 / 8$; $P=0.073)$. Since the validity factor did not interact significantly with $\operatorname{SOA}(F=1.93$; $d f=6 / 24 ; P<0.11)$ or with cue modality $(F=1.28 ; d f=2 / 8 ; P<0.330)$, there were also no significant costs or benefits associated with particular values of these factors.
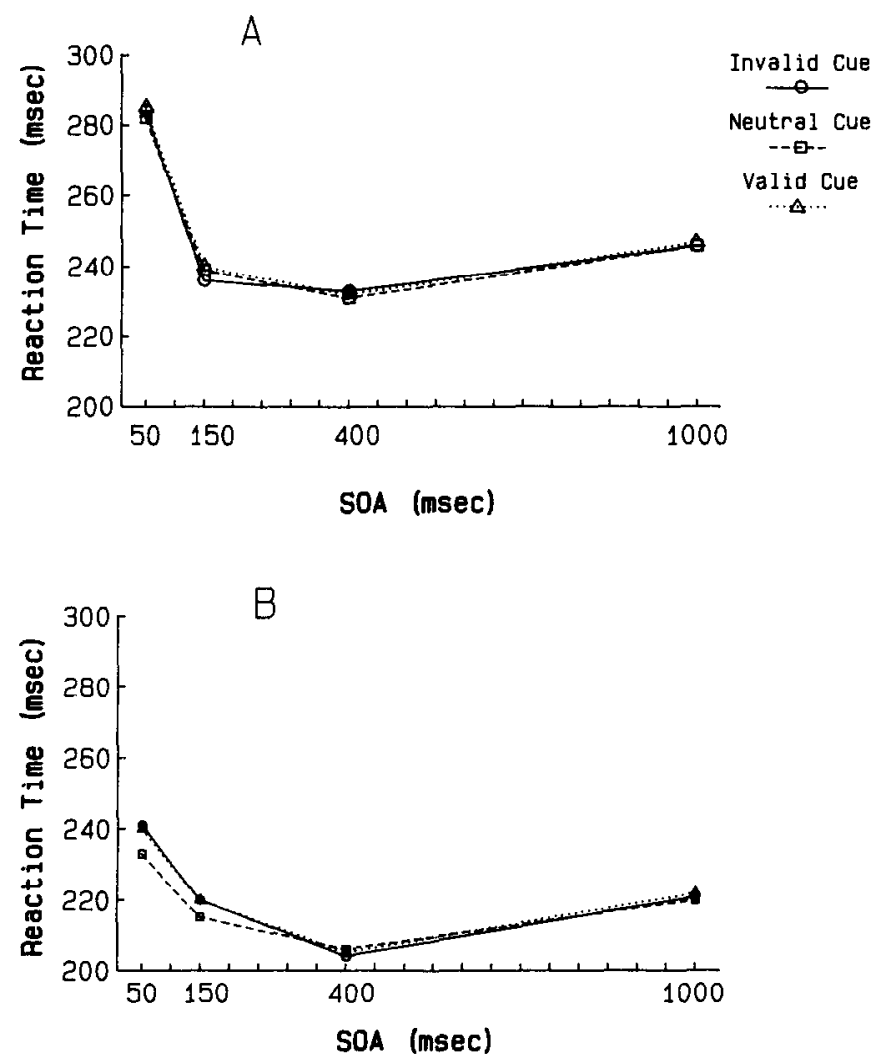

Fir. 4. Mean reaction times to the auditory target for the four SOAs and the three validity conditions. (A) Auditory cues. (B) Visual cues. 
It is clear from Fig. 4 that SOA affected performance when either cue was presented. As in Experiment 1, RTs declined from 50 to 150 and from 150 to $400 \mathrm{msec}$ and then increased somewhat at the $1000 \mathrm{msec}$ SOA. Consequently, the effect of SOA was significant $(F=10.87$; $d f=3 / 12 ; P=0.001$ ). However, comparisons of RTs at $50 \mathrm{vs} 150 \mathrm{msec}$, or $50 \mathrm{vs} 400 \mathrm{msec}$, the values between which the largest $R T$ decrement was found, did not attain significance when cvaluated by two-tail $t$-tests with the confidence interval adjusted for multiple comparisons $(P<0.017)$.

Furthermore, RTs on trials with the visual cue were consistently shorter than RTs on trials with the auditory cue at all SOAs (see Fig. 4). This difference, which on the average was $30 \mathrm{msec}$, yielded a highly significant cue modality effect $(F=36.38 ; d f=1 / 4 ; P=0.004)$. In addition, the cue modality $\times \mathrm{SOA}$ interaction was significant $(F=4.58 ; d f=3 / 12$; $P=0.0239$ ), reflecting the finding that the RT decrement associated with the visual cue was greater than the RT decrement associated with the auditory cue from 50 to $150 \mathrm{msec}$, but not at other values of SOA. However, this difference in the RT decrements associated with the two cues was not reliable when evaluated by a two-tail $t$-test (confidence interval adjusted to $P<0.017$ ).

In addition to the significant main effect of cue modality described above, one other factor-side of target presentation--was significant $(F=17.72 ; d f=1 / 4 ; P=0.014)$, reflecting the finding that RTs to targets on the left $(\bar{X}=233.8 \mathrm{msec})$ were shorter than RTs to targets on the right $(\bar{X}=237.3 \mathrm{msec})$.

Finally, two higher-order interactions attained significance: side of target presentation $\times$ cue validity $\times \operatorname{SOA}(F=3.75 ; d f=6 / 24 ; P=0.009)$ and cue modality $\times$ side of target presentation $\times \mathrm{SOA} \times$ experience $(F=3.54 ; d f=3 / 12 ; P=0.048)$. The former interaction was apparently due to the relatively greater RT decrement from the $50 \mathrm{msec}$ to the $150 \mathrm{msec}$ SOA and the relatively smaller RT decrement from the 150 to the $400 \mathrm{msec}$ SOA when the target was presented on the left side compared to the right side. The latter interaction was apparently due to the relatively greater RT decrement from the $50 \mathrm{msec}$ to the $150 \mathrm{msec} \mathrm{SOA}$ of unexperienced subjects when the target was presented on the right compared to the left side.

\section{DISCUSSION AND CONCLUSIONS}

The field effect found in Experiment 2 (RTs to leftward auditory targets being $3.5 \mathrm{msec}$ faster than to rightward auditory targets) was opposite to the field effect found in Experiment 1, where the target was visual (4.4 msec advantage for right visual field stimuli). Since there was no interaction between the field effect found in Experiment 2 and cue modality, this effect apparently depends solely upon the target's auditory modality and may reflect faster spatial processing of auditory stimuli by the right hemisphere. Apparently this differential processing efficiency of the right hemisphere outweighs the advantage, found in Experiment 1 , conferred by compatibility of hand and ficld.

In Experiment 2, as in Experiment 1, a cue in the same modality as the target was consistently associated with longer RTs than was a cue in a modality different from that of the target. Since this effect was found in both experiments, it is not modality specific. Rather, as suggested previously, it may show that inhibition of responses to the cue was still operating at the time the target was presented and interfered with responses to targets in the same modality. Alternatively, it may reflect the limited capacity of a modality-specific channel [3]. One or the other of these factors may also account for differences in the slopes of the RT-SOA functions obtained in both experiments. In Experiment 2, the auditory cue was associated 
with a greater RT decrement from 50-150 msec than was the visual cue; in Experiment 1, a greater RT decrement was found with the visual cue than with the auditory cue at these same SOA values. These two findings might also be accounted for by slower processing of targets in the same modality as the cue compared to targets in a different modality. According to this interpretation, at short SOAs the target arrives before processing of the cue is complete, whereas with long SOAs the processing is already done by the time the target arrives so a response can follow quickly.

The major finding of Experiment 2 was that the same visual and auditory cues that led to costs and benefits when the target was visual (Experiment 1 ) were totally ineffective when the target was auditory. The only effect of the cues in Experiment 2 was an RT advantage of the neutral cue relative to the other two cues, and even this effect was small and of marginal significance. This finding may reflect an alerting effect of the neutral cue, which, unlike the valid and invalid cues, consisted of two stimuli; this presumed effect only became apparent in the absence of the much stronger effect of cue validity found in Fxperiment 1 . The failure to find costs or benefits of spatial cues on RTs to auditory stimuli is consistent with a similar negative finding [10]; in that study, visual symbolic cues also had no effects on simple RTs to auditory or tactile stimuli. However, there is evidence that auditory cues can under certain conditions lead to costs or benefits in RTs to auditory stimuli. MAzZUCCHI et al. [8] found a small cost $(5-6 \mathrm{msec})$ of invalid verbal auditory cues on RTs to monaural clicks, but no benefit when the same cues were valid. They also reported a small benefit $(6-7 \mathrm{msec})$ of valid non-verbal auditory cues (musical tones presented binaurally) on RTs to monaural clicks, but no costs when the same cues were invalid. It is unlikely that our failure to find costs or benefits like those reported by Mazzucchi et al. when auditory cues and targets were presented in Experiment 2 is attributable to the nature of the cues we used, for, as mentioned above, these auditory cues were effective in Experiment 1. It is possible that the findings of Mazzucchi et al. depended upon their use of monaural target stimuli. There is evidence that attentional biases can influence the effect of a monaural auditory input on event-related potentials [6]. In the case of our binaural, spatially-displaced auditory stimuli, localization, as is normally the case, depended on the difference in time of arrival of the two ears. It is not likely that the absence of costs and benefits in Experiment 2 was due to a difficulty in localizing the auditory target. First, the subjects reported that they had no difficulty in localizing the auditory target in the practice session of Experiment 2 . Second, the auditory target was similar to the auditory cue, which was an effective spatial cue in Experiment 1, and thus must have been correctly localized in that experiment.

It should be pointed out that the neutral cue we used may have led to facilitatory and/or inhibitory effects on RT because it involved bilateral presentation of lateralized stimuli, that is, stimuli containing salient spatial information. In the case of visual stimuli, at least, the subjects may have attended randomly to one or the other of the two peripheral cues. This lateral shift of attention would be unlikely with the neutral auditory cues because most subjects experienced the cue as a single sound source coming from a central location (stereophonic fusion). The possibility that the peripheral neutral cue led to inhibitory effects at long SOAs [7, 11] seems unlikely, for we did not find inhibitory effects of valid peripheral cues at these SOAs. Furthermore, validity effects were still very clear with the visual target at the long SOAs.

The finding that the same visual cue yielding costs and benefits when a visual target is employed is ineffective when an auditory target is employed invalidates our hypothesis that cues in a modality different from the target are as effective as cues in the same modality in 
covertly shifting attention. The finding raises the question: what special features are possessed by visual stimuli (but not by auditory stimuli) that allows covert orienting to them by cues in the same or different modalities? One possible answer to this question is that visual stimuli, unlike auditory stimuli, typically evoke orienting eye and head movements that improve stimulus identification because the foveal region of the retina can be brought to bear on stimuli needing fine analysis. According to this view, when the task requires responses to visual targets, as in Experiment 1, altention will shift covertly irrespective of the cue modality because the cues are linked to a system (i.e. vision) that controls orienting movements for improving identification of the target stimulus (even though overt orienting was prevented in our situation); with auditory targets, as in Experiment 2, no covert orienting would be evoked by the same auditory and visual cues effective in Experiment 1 because there are no orienting movements of the auditory system that serve to improve identification of auditory stimuli (orienting movements of the head may improve auditory localization but there is no equivalent of the fovea in the auditory system). It should be noted that looking at the source of spoken words improves their recall in a verbal memory test [13], suggesting that under particular circumstances the visual orientation system can influence auditory attentional mechanisms.

This interpretation of our findings in terms of sensory receptor gradients leads to the prediction that a tactile cue would be as effective as a visual or auditory cue in covertly shifting attention to visual stimuli. Furthermore, according to our analysis, one would expect that non-tactile as well as tactile cues would be effective in covertly shifting the attention to the tactile target, for tactile stimuli can evoke orienting body movements (e.g. of the hands) that improve the identification of tactile stimuli by bringing maximally sensitive portions of the skin (e.g. the finger tips) onto the stimulus. We are currently testing these predictions.

Acknowledgements - Supported, in part, by a grant to $\mathrm{HAB}$ from the Veterans Administration Department of Medicine and Surgery. Manuscript preparation was aided by the Departments of Psychiatry and Psychology, University of Michigan. We thank Bob Zuckernik and Dean Galatis for programming and help in constructing the apparatus, Richard Santucci and Dean Galatis for running subjects, and Patrick Brown for helpful comments on the manuscript.

\section{REFERENCES}

1. Anzola, G. P., Bertoloni, G., Buchtel, H. A. and Rizzolatti, G. Spatial compatibility and anatomical factors in simple and choice reaction time. Neuropsychologia 15, $295-302,1977$.

2. BAShinski, H. S. and Bacharach, V. R. Enhancement of perceptual sensitivity as the result of selectively attending to spatial locations. Percept. Psychophys. 28, 241-248, 1980.

3. Broniderest, D. E. Perception and Commanication. Pergamon, New York, 1958.

4. Bitter, C. M. The role of polysensory neuronal systems in spatially-directed attention and orienting responses to external stimuli. In Attention: Theory, Brain Function and Clinical Application. D. SHEER and K. PriBraM (Fditors), Academic Press. New York. in press.

5. ERicksen, C. W. Partitioning and saturation of visual displays and efficiency of visual search. J. appl. Psychol. 39, $7377,1955$.

6. Hillyari, S. A., Hink, R. F., Schwist, V. L. and Picton, T. W. Electrical signs of selective attention in the human brain. Science 182, 177180,1973

7. MAYIOR, F. A. and HOCKLY, R. Inhibitory component of externally controlled covert orienting in visual space. I. exp. Psychol. (Human Percept.) 11, 777 787. 1985.

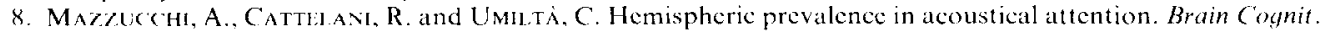
$2,111,1983$.

9. Poflinblikgr, A. T. Reaction time to retinal stimulation with special reference to the time lost in conduction through nerve centers. Archs Psichol. 23, 1 73, 1912.

10. Posner, M. I. Chronometric Explorations of the Mind. Erlbaum. Hillsdale. New Jersey. 1978 
11. Posner, M. I. and Cohen, Y. Components of visual orienting. In Attention and Performance X, H. Bauma and D. Bowhuis (Editors), pp. 531-556. Erlbaum, Hillsdale, New Jersey, 1984.

12. Posner, M. I., Snyder, C. R. and Davidson, B. J. Attention and the detection of signals. J.exp. Psychol. 109, $160-174,1980$.

13. Reisberg, D., Scheiber, R. and Potemken, L. Eye position and the control of auditory attention. $J$. exp. Psychol., Hum. Percept. Perform. 7, 318-323, 1981.

14. Remington, R. Attention and saccadic eye movements. J. exp. Psychol. 6, 726-744, 1980.

15. Sperling, G. and Melchner, M. J. The attention operating characteristics: examples from visual search. Science 202, 315 318, 1978. 